\title{
Water-window microscopy using a compact, laser-plasma SXR source based on a double-stream gas-puff target
}

\author{
Przemyslaw W. Wachulak • Andrzej Bartnik • Marcin Skorupka • \\ Jerzy Kostecki • Roman Jarocki • Mirosław Szczurek • Lukasz Wegrzynski • \\ Tomasz Fok $\cdot$ Henryk Fiedorowicz
}

Received: 28 August 2012/Accepted: 23 December 2012/Published online: 2 February 2013

(C) The Author(s) 2013. This article is published with open access at Springerlink.com

\begin{abstract}
We report on a compact, desktop size, laboratory type microscopy setup, based on a short wavelength gas puff target soft X-ray source, which emits incoherent radiation in "water-window" spectral range. The microscope employs a Wolter type I reflective objective and allows capturing magnified images of objects with $\sim 1-\mu \mathrm{m}$ spatial resolution and exposure time as low as $5 \mathrm{~s}$. A detailed characterization and optimization of both the source and the microscope setups are presented and discussed.
\end{abstract}

\section{Introduction}

Decreasing the illumination wavelength is a direct way to improve spatial resolution in photon-based imaging systems, thus the widespread interest in microscopy at extreme ultraviolet (EUV) and even shorter, soft X-ray (SXR) wavelengths. Utilizing coherent illumination a $700 \mathrm{~nm}$ half-pitch resolution images with EUV recombination laser at $\lambda=18.2 \mathrm{~nm}$ has been reported in the early imaging work [1]. Better spatial resolution equal to $75 \mathrm{~nm}$ was reported utilizing an SXR laser at $\lambda=4.48 \mathrm{~nm}$, pumped by a large fusion class NOVA laser [2]. Recently different approaches have emerged due to the development of smaller scale short-wavelength sources such as HHG [3],

P. W. Wachulak $(\bowtie) \cdot$ A. Bartnik · J. Kostecki · R. Jarocki · M. Szczurek · L. Wegrzynski · T. Fok · H. Fiedorowicz Institute of Optoelectronics, Military University of Technology, ul. gen. S. Kaliskiego 2, 00-908 Warsaw, Poland

e-mail:wachulak@gmail.com

M. Skorupka

Military University of Technology, ul. gen. S. Kaliskiego 2, 00-908 Warsaw, Poland
EUV lasers [4] and incoherent laser plasma-based sources [5] that have been successfully used for sub-micrometer resolution imaging. Using radiation from a table-top capillary discharge EUV laser images were obtained with a spatial resolution of $120-150 \mathrm{~nm}$ [6]. $\lambda=13.2 \mathrm{~nm}$ wavelength radiation from $\mathrm{Ni}$-like cadmium EUV laser allowed for a sub-38 nm resolution nano-imaging [7]. A quasimonochromatic emission from a laser-plasma EUV source based on a gas puff target [8] allowed for sub-70 nm spatial resolution imaging $[9,10]$ in a very compact system. A quasi-monochromatic emission from an incoherent SXR source, at much shorter wavelength, $\lambda=2.88 \mathrm{~nm}$, in the "water-window" spectral range, based on the liquid nitrogen, allowed to demonstrate SXR microscopy with a sub-50 nm spatial resolution [11]. Ethanol droplet-based SXR source at $\lambda=3.37 \mathrm{~nm}$, combined with a zone plate objective allowed to capture images with spatial resolution of $60 \mathrm{~nm}$ [12], later improved to $\sim 50 \mathrm{~nm} \mathrm{[13].} \mathrm{Liquid}$ nitrogen-based SXR source at $\lambda=2.48 \mathrm{~nm}$ was used to demonstrate recently a compact full-field soft X-ray transmission microscopy with sub-60 nm resolution, operating at $100 \mathrm{~Hz}$ repetition rate, with exposure times of less than $5 \mathrm{~min}$ using both dry and wet samples [14]. Tomographic high resolution imaging was also performed in the "water-window" spectral range with a diatom, acquiring a tilt series of 53 images covering $180^{\circ}$ with halfperiod spatial resolution of the tomogram approaching $140 \mathrm{~nm}$ [15]. Using a Schwarzschild reflective objective, with $32 \times$ magnification and $\mathrm{NA}=0.2$ images of test objects were acquired with a half-pitch spatial resolution better than $0.5 \mu \mathrm{m}$ [16].

Large synchrotron facilities were also extensively utilized for the implementation of full field microscopes with record spatial resolution of 12 [17] or $14 \mathrm{~nm}$ utilizing $\lambda=1.38 \mathrm{~nm}$ undulator radiation and third order zone plate 
diffraction [18], also including magnetic material imaging [19-22]. Soft X-ray (SXR) microscopy has been successfully employed mainly in transmission mode, either using diffractive optics, such as zone-plates [23-25], raster scanning of the sample by focused SXR beam [26-28] or as a contact microscopy, where the sample is placed on top of a recording medium, such as a photoresist, and illuminated by SXR beam to make a "picture" of the specimen in the surface of the recording medium [29-31]. Synchrotron radiation at $\lambda=2.4 \mathrm{~nm}$ was used for imaging frozenhydrated samples at atmospheric pressure, where details inside cells of algae as small as $35 \mathrm{~nm}$ were visible [32], or to examine rapidly frozen mouse 3T3 cells and obtained excellent cellular morphology at better than $50 \mathrm{~nm}$ lateral resolution, using transmission SXR microscope [33]. Synchrotron-based microscope in the "water-window" spectral range was developed to image frozen hydrated specimens with a thickness of up to $10 \mu \mathrm{m}$ at temperatures of around $100 \mathrm{~K}$ [34].

Photon-based (bosonic-type) imaging at short wavelength versus electron, or recently neutron, imaging has additional advantages due to different interaction of photons with matter. Atomic resonance frequencies, leading to very high absorption coefficients at EUV and SXR wavelengths, provide an enhanced optical contrast. Incoherent imaging techniques are somewhat complimentary to coherent ones. Although the optical transfer function (OTF) for incoherent illumination is capable to transfer two times higher spatial frequencies, than for the case of coherent illumination, the incoherent type of illumination often requires the use of additional optics, such as zone plate lenses. In that case the spatial resolution of, for example, zone plate microscopy, might be inferior to coherent imaging techniques, which often does not require any additional optics, but the coherence of the source can be problematic due to coherence effects present in the image, such as "twin-image" problem in holography. This is not the case for incoherent illumination, exploited in this work.

Particularly suitable range of wavelengths for biological imaging is so-called the "water-window" spectral range. $\mathrm{X}$-ray sources, emitting in the "water-window" region between 2.3 and $4.4 \mathrm{~nm}$ wavelength [35], are thus important for biological applications. High contrast in this spectral range is obtained due to a difference in absorption of different constituents of biological specimen. While water, present in the sample, has relatively small absorption coefficient in this spectral range, carbon, due to much higher absorption, gives very good contrast in the image. Thus, this spectral range is perfectly suitable for imaging of biological specimen.

In this work we try to combine the advantages of employing compact, laboratory type laser-plasma short wavelength source, based on a gas puff target, emitting incoherent radiation, with the "water-window" spectral range. This unique combination is suitable for biological imaging, and allows in developing a small size microscopy setup, which might be used in various fields of science and technology. Thus, in this paper, we report for the first time on "water-window" compact, desktop microscopy setup employing a laser-plasma SXR source based on a doublestream gas-puff target and Wolter type I objective. The system allows capturing magnified images of the objects with $\sim 1 \mu \mathrm{m}$ spatial resolution and exposure time as low as $5 \mathrm{~s}$. A detailed characterization and optimization of both the source and the microscope setup are presented.

\section{SXR source description and microscope experimental setup}

The "water-window" microscope was equipped with an ellipsoidal SXR condenser coated with nickel, to focus SXR radiation onto an object. A Wolter type I reflective objective was used to form a magnified image onto an SXR-sensitive CCD (charge coupled device) camera in transmission mode. The use of the gas puff target eliminates debris production problem associated with solid targets. Radiation from the "water-window" spectral range was selected by a titanium filter. Test objects- two distinct patterns of copper meshes were imaged with a half-pitch spatial resolution approaching $1 \mu \mathrm{m}$ in a very compact set up and with short exposures. The experimental setup of the SXR microscopy system is shown in Fig. 1.

The EUV source, used in the experiment, has been developed for EUV metrology applications in the frame of MEDEA+ project [36] and later modified for efficient emission of SXR radiation, including the "water-window" spectral region, previously reported in [37]. This source has an advantage over other compact sources that it is a debrisfree source and has a possibility to change the working gases, thus allowing to change both the peak emission wavelength and the inverse relative bandwidth of the emission.

For the SXR microscope Ar plasma was produced by focusing of the pumping laser pulses, from Nd:YAG laser (Eksma), with duration of $4 \mathrm{~ns}$ and energy of $0.74 \mathrm{~J}$ by an $f=25 \mathrm{~mm}$ focal length lens onto a gas puff target. The plasma radiates in a very broad range of wavelengths, including SXR region and by using additional spectral filtering it is possible to tailor the spectral emission of the source. The source can operate up to $10 \mathrm{~Hz}$ repetition rate. A pressure of $1.5 \times 10^{-4}$ mbar was constantly maintained in the microscope chamber during the source operation. The experimental setup is extremely compact. The microscope is located inside a vacuum chamber, $60 \mathrm{~cm}$ in 
Fig. 1 (color online) Scheme of the system (a), photograph with indicated components (b)
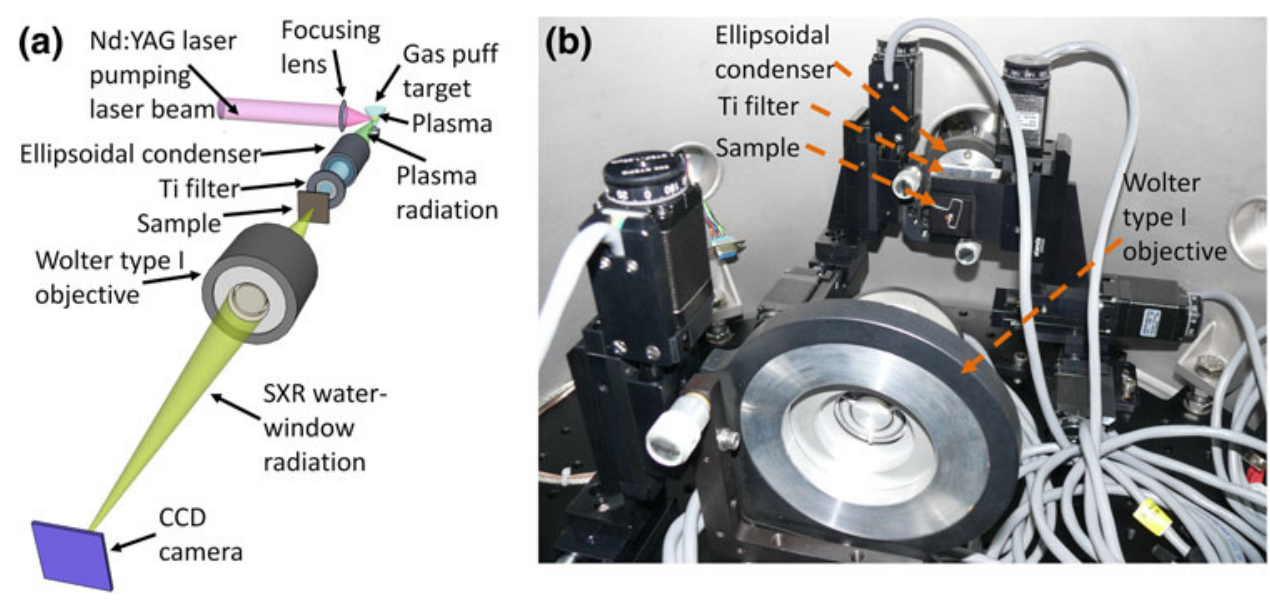

diameter and $35 \mathrm{~cm}$ in height and the entire system fits on top of a single $1.8 \times 1.2 \mathrm{~m}^{2}$ optical table.

EUV radiation from the plasma was collected and focused by an ellipsoidal, axi-symmetrical nickel coated condenser mirror, developed by Rigaku, Inc. The condenser is a broad-band optic, capable of efficiently reflecting radiation from the EUV range down to SXR region with energy cut-off of $\sim 600 \mathrm{eV}(\sim 2.06 \mathrm{~nm}$ critical wavelength). The distance between foci of the condenser was equal to $270 \mathrm{~mm}$. The distance from the plasma, positioned in the first focal plane to an entrance plane of the condenser was $140 \mathrm{~mm}$, while the distance from an exit plane to the second focal plane- $60 \mathrm{~mm}$. Entrance diameter was equal to $14 \mathrm{~mm}$ and results in entrance numerical aperture of $\mathrm{NA}_{\mathrm{C}_{-} \text {in }}=0.05$, while the exit diameter of $11.7 \mathrm{~mm}$ corresponds to exit numerical aperture of $\mathrm{NA}_{\text {C_out }}=0.09$.

The laser plasma source was optimized for efficient generation of SXR radiation from argon plasma. The target is formed by two circularly symmetric nozzles. The inner nozzle, $0.4 \mathrm{~mm}$ in diameter, injects a small amount of working gas (argon) into the vacuum, $250 \mu$ s after arrival of synchronization pulse from laser power supply. The nozzle stays open for $600 \mu \mathrm{s}$. The outer nozzle, ring-shaped $0.7-1.5 \mathrm{~mm}$ in diameter, injects a small Z-number gas, in our case helium, to narrow down the flow of the working gas, reducing its density gradient along the normal to the nozzle axis. The nozzle opens $750 \mu$ s after arrival of the synchronization pulse and stays open for $200 \mu \mathrm{s}$. A $50 \mu \mathrm{s}$ later the laser pulse is generated producing in turn an SXR pulse. The nozzle axis was positioned almost concentrically with the laser focal point, displaced $0.1 \mathrm{~mm}$ in the direction opposite to the condenser optic, to reduce the absorption of SXR radiation in a neutral gas from the target. The distance from the focal point to the nozzle plane was $1.5 \mathrm{~mm}$ to avoid nozzle damage by plasma formation. The details of the optimization were similar to one described previously in [37].
To spectrally narrow the emission from argon plasma a $200 \mathrm{~nm}$ thick, $10 \mathrm{~mm}$ in diameter, free-standing titanium filter (Lebow) was used, positioned $21 \mathrm{~mm}$ downstream the condenser. Spectrally filtered radiation illuminates the sample, positioned $60 \mathrm{~mm}$ downstream the condenser, in its second focal point. Then, the sample is imaged onto an SXR sensitive back-illuminated, $1,024 \times 1,024$ pixels, $13 \times 13 \mu \mathrm{m}^{2}$ pixel size, CCD camera (i-Kon, DO-934N model, from Andor) by a Wolter type I reflective objective [38]. The objective is composed of two axially symmetric ellipsoidal and hyperboloidal nickel coated mirrors. The object plane of the objective, which coincides with the sample plane, is located $\sim 150 \mathrm{~mm}$ from its entrance aperture, $15 \mathrm{~mm}$ in diameter with $14 \mathrm{~mm}$ central beam stop. The image plane is $\sim 2,190 \mathrm{~mm}$ from the objective's exit aperture, $17 \mathrm{~mm}$ in diameter with $15.5 \mathrm{~mm}$ diameter central beam stop. The magnification of the objective is thus equal to $14.6 \times$ and the image pixel size is $890 \times 890 \mathrm{~nm}^{2}$. The entrance numerical aperture of the objective is equal to $\mathrm{NA}_{\mathrm{O}_{-} \text {in }}=0.05$, while the exit numerical aperture- $-\mathrm{NA}_{\mathrm{O}_{-} \text {out }}=0.0038$. Exit numerical aperture of the condenser $\left(\mathrm{NA}_{\mathrm{C}_{-} \text {out }}=0.09\right)$ is almost twice

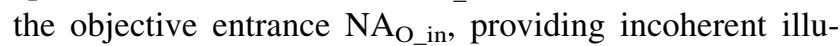
mination [39], since $\sigma=\mathrm{NA}_{\mathrm{C} \_ \text {out }} / \mathrm{NA}_{\mathrm{O} \_ \text {in }}=1.8$.

The condenser, sample stage and objective were mounted on three axis translation stages driven by vacuum compatible step motor actuators (Standa). To obtain a single image in the "water-window" spectral range 50-100 SXR pulses were necessary, at $10 \mathrm{~Hz}$ repetition rate. During image acquisition the CCD camera was cooled down to $-10{ }^{\circ} \mathrm{C}$ to decrease the intrinsic, thermal noise of the detector.

\section{Source measurements}

Measurements of the source photon flux were performed using commercial AXUV100 silicon p-n junction photodiode, 
from International Radiation Detectors, Inc., and corrected for transmission of the Ti filter. Measured number of photons was equal to $1.3 \times 10^{11}$ photons/sr/pulse or $1.6 \times 10^{12}$ photons in a $4 \pi$ solid angle per SXR pulse, in the transmission band of the filter (from 2.8 to $\sim 6 \mathrm{~nm}$ wavelength), assuming a uniform angular distribution of emitted photons. Subsequent measurements yielded the number of photons in the focal plane of the condenser, equal to $3.6 \times 10^{10}$ photons/pulse, which corresponds to $2.3 \mu \mathrm{J} /$ pulse in band.

The SXR spectra, obtained with and without the filter have been measured using a transmission grating spectrometer equipped with 5,000-lines/mm free-standing grating, $33 \mu \mathrm{m}$ entrance slit and a CCD camera (i-Kon). The grating was positioned $\sim 680 \mathrm{~mm}$ from the plasma, the entrance slit was placed $4 \mathrm{~mm}$ from the grating and distance between the grating and the CCD was $145 \mathrm{~mm}$. From geometry of the spectrometer and the grating, the wavelength range was up to $16 \mathrm{~nm}$. The inverse relative bandwidth (IRB) of the spectrometer was estimated from the spectrum to be $\lambda / \Delta \lambda \sim 50$ at $4 \mathrm{~nm}$ wavelength. The spectrum of Ar plasma, both direct and filtered by Ti filter is shown in Fig. 2a. The spectrum obtained without the filter required only $5 \mathrm{SXR}$ pulses, while the one obtained with the filter required 100 SXR pulses. Figure 2b, c shows raw data from the CCD camera for both spectra. The figure depicts Ar emission spectrum in the wavelength range from
2 to $16 \mathrm{~nm}$. The dominant groups of argon spectral lines are $\mathrm{Ar}^{8+}: 2 \mathrm{~s}^{2} 2 \mathrm{p}^{6}-2 \mathrm{~s}^{2} 2 \mathrm{p}^{5} 4 \mathrm{~s}$, wavelengths between $\lambda=$ $36.78 \AA$ and $\lambda=36.96 \AA, \mathrm{Ar}^{8+}: 2 \mathrm{~s}^{2} 2 \mathrm{p}^{6}-2 \mathrm{~s}^{2} 2 \mathrm{p}^{5} 3 \mathrm{~d}(\lambda=$ 41.48-42.56 $\AA$ ), $\mathrm{Ar}^{8+}: 2 \mathrm{~s}^{2} 2 \mathrm{p}^{6}-2 \mathrm{~s}^{2} 2 \mathrm{p}^{5} 3 \mathrm{~s}(\lambda=48.73-49.18$ $\AA)$. In the longer wavelength range, filtered out by Ti filter, the dominant spectral groups are: $\mathrm{Ar}^{7+}: 2 \mathrm{p}^{6} 3 \mathrm{~s}-2 \mathrm{p}^{6} 5 \mathrm{p}(\lambda=$ 120.09-120.16 $\AA$ ), $\mathrm{Ar}^{7+}: 2 \mathrm{p}^{6} 3 \mathrm{p}-2 \mathrm{p}^{6} 6 \mathrm{~d}(\lambda=122.62-123.03$ $\AA)$ and $\mathrm{Ar}^{7+}: 2 \mathrm{p}^{6} 3 \mathrm{p}-2 \mathrm{p}^{6} 5 \mathrm{~d} \quad(\lambda=137.93-138.44 \AA)$ according to the data reported in [40]. Most of the spectrally filtered radiation is from the "water-window" spectral range, usually defined as the wavelength range from 2.3 to $4.4 \mathrm{~nm}$.

A spatial distribution of the Ar plasma in the "waterwindow" spectral range was obtained using a pinhole camera. The measurements were performed with Ti filter, to assess the plasma size particularly in the "water-window" range. Laser drilled, $32 \mu \mathrm{m}$ in diameter pinhole was positioned $291 \mathrm{~mm}$ from the plasma and $280 \mathrm{~mm}$ from the CCD camera (X-vision M25, Reflex s.r.o., Czech Republic), which was equipped with $512 \times 512$ pixels CCD chip, $0.5 \times 0.5 \mathrm{in}^{2}$ in size. This results in a lateral magnification of $0.96 \times$.

The spatial distribution of argon plasma in the "waterwindow" spectral range is depicted in Fig. 3a and crosssections in vertical and horizontal directions are shown in Fig. 3b. For the measurements 300 SXR pulses were
Fig. 2 (color online) Spectrum of the SXR radiation (a) and CCD data without (b) and with (c) Ti $200 \mathrm{~nm}$ filter, respectively

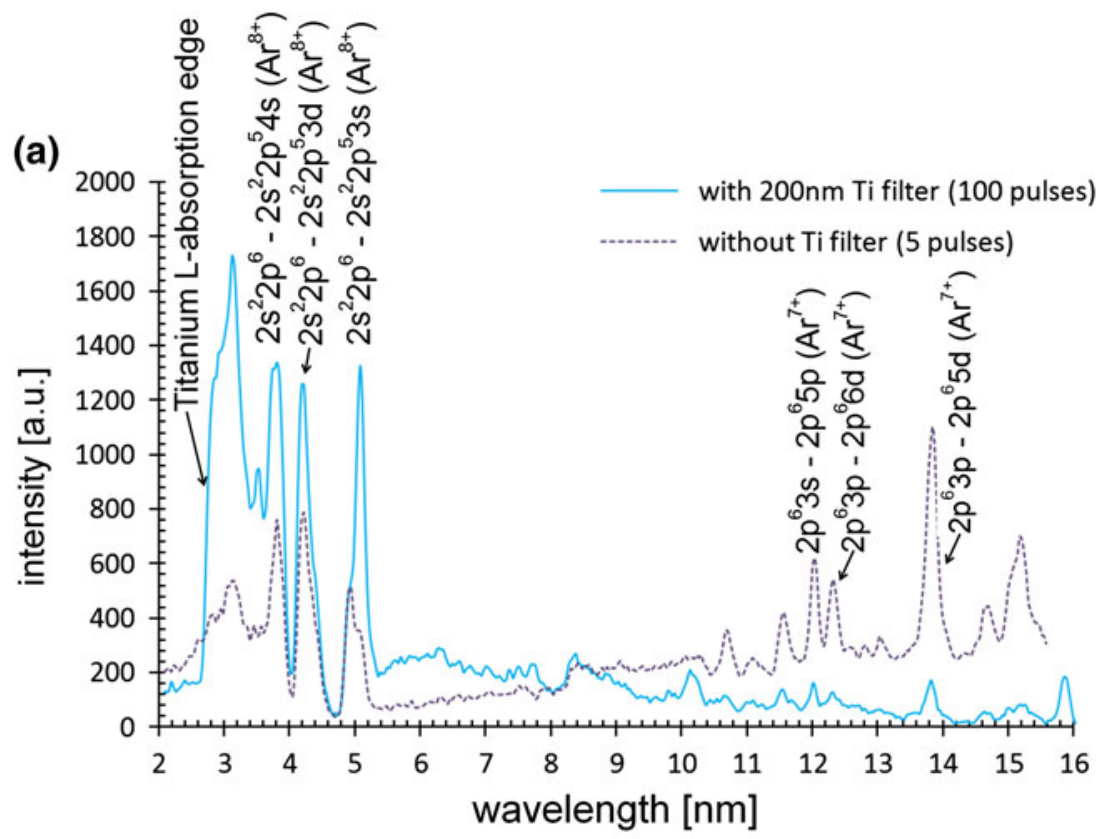

(b)

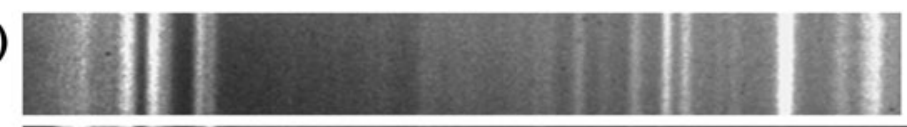

(c)

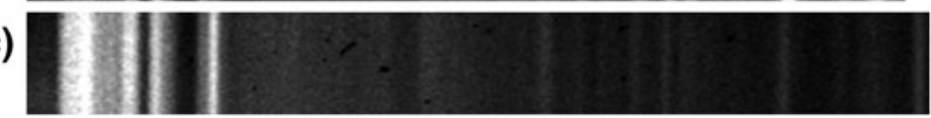



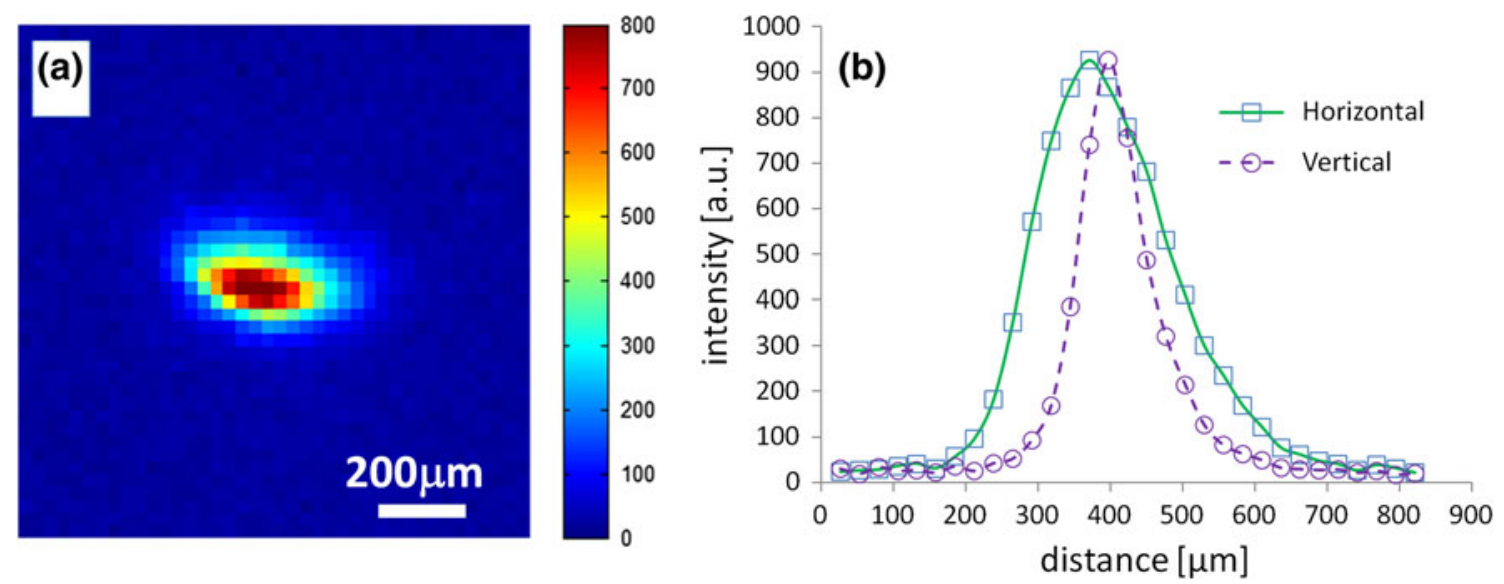

Fig. 3 (color online) Pinhole camera image of plasma spatial intensity distribution a and cross-sections in horizontal and vertical directions (b)

required. The argon plasma FWHM size was measured to be $240 \times 130 \mu \mathrm{m}^{2}$.

To illuminate the sample uniformly a proper condenser alignment was necessary. For that a small beam block, $\sim 5 \mathrm{~mm}$ in diameter, was placed at the exit plane of the condenser, to block a direct light from the plasma. Radiation, focused by the condenser, was then scattered by a $750 \mathrm{~nm}$ thick Al film placed in the vicinity of the focal plane of the condenser. Scattered light was imaged on to a CCD camera (i-Kon, Andor) by previously mentioned pinhole camera, to form a slightly magnified image of the spatial distribution of light downstream the condenser near its focal plane. The distance from the $\mathrm{Al}$ foil to the pinhole was equal to $360 \mathrm{~mm}$ and from the pinhole to the detector- $-325 \mathrm{~mm}$, resulting in lateral magnification of $1.11 \times$. The optimization of the condenser position was performed and the examples of intensity distributions of scattered radiation from the $\mathrm{Al}$ foil, focused by the condenser optic, for different condenser position are shown in Fig. 4. If the condenser is properly aligned, the intensity distribution is circularly symmetric and has smallest area, as shown in Fig. 4b. However, if the optic is misaligned the images of the focal plane show asymmetry and the spot is bigger, yielding lower photon density at the focal plane, Fig. $4 \mathrm{a}$, c. The numbers, shown for each figure, correspond to a distance of the condenser from its optimal position. In this example, the condenser was misaligned in the plane perpendicular to the optical axis of the system.

\section{4 "Water-window" microscope imaging results}

As test objects, two transmission electron microscope (TEM) grids (meshes) (Tesla, Czech Republic) of different geometries were used. Typical scanning electron microscope (SEM) images of the objects are shown in Fig. 5. A square-shaped mesh, shown in Fig. 5a, has a period of $(123.8 \pm 0.5) \mu \mathrm{m}$ and a bar with width $(42.9 \pm 0.7) \mu \mathrm{m}$,
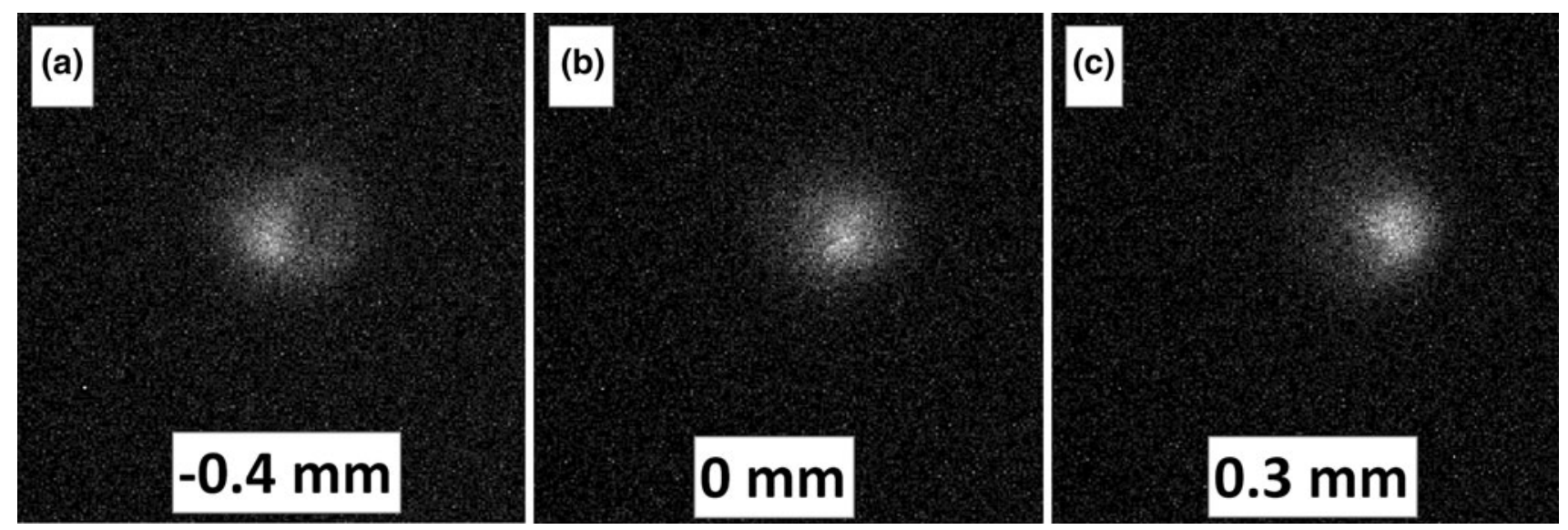

Fig. 4 Intensity distribution of the radiation, obtained by scattering from $750 \mathrm{~nm}$ thick $\mathrm{Al}$ foil, focused by an ellipsoidal condenser if properly aligned (b) and for slightly misaligned condenser in direction perpendicular to the optical axis, around the optimal position (a, c). Numbers indicate the amount of misalignment (distance) 
Fig. 5 SEM images of the objects: square mesh (a) and rectangular mesh (b)
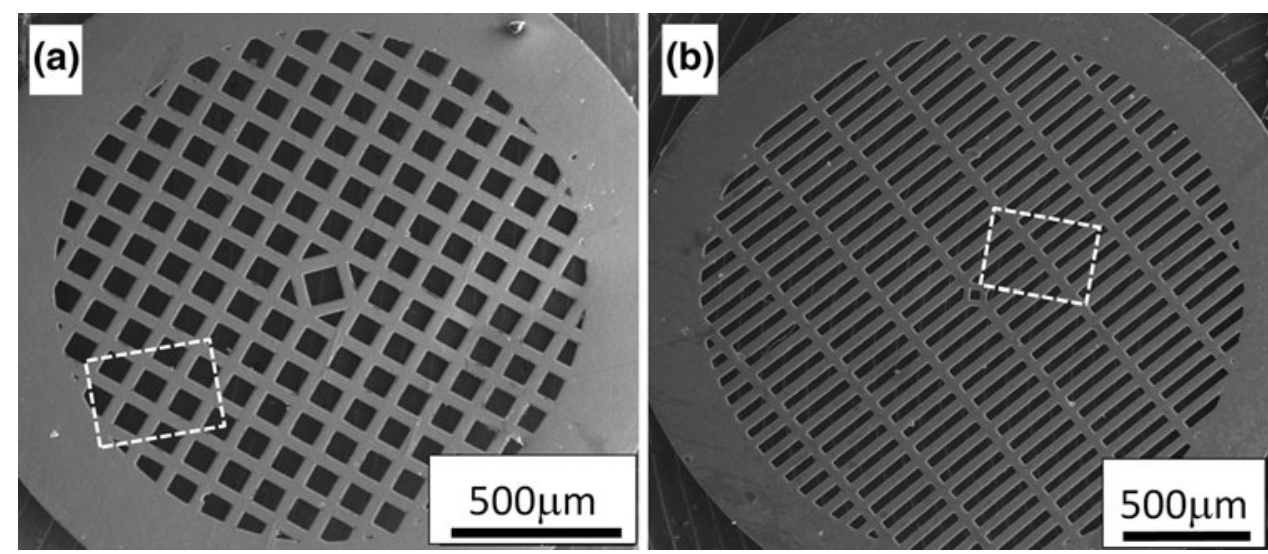

while a rectangular mesh, shown in Fig. 5b, has a period of $(83.2 \pm 1.4) \mu \mathrm{m}$ and a slit width of $(37.1 \pm 0.8) \mu \mathrm{m}$. The errors are associated with the accuracy of grating fabrication. The thickness of both grids was $\sim 13 \mu \mathrm{m}$. Dashed boxes indicate the regions of the sample imaged with the "water-window" microscope.

To obtain the sharpest possible image in the "waterwindow" spectral range, series of images was recorded at various sample-objective distances, in the range of $\sim \pm 2 \mathrm{~mm}$ from the focal point of the objective. From the entire set of images, the "sharpest" SXR image was chosen for subsequent resolution measurements. Resolution of the microscope was assessed by a well established knife edge (KE) test. For incoherent illumination the 10-90 \% intensity transition across a sharp edge corresponds to a well known Rayleigh resolution and to twice the value of halfpitch grating resolution of the optical system [41].

Typical images of the meshes, obtained under argon plasma illumination, filtered by $200 \mathrm{~nm}$ thick Ti filter are shown in Fig. 6a, for square mesh and b for a rectangular mesh, respectively. Black dotted line, depicted in Fig. 6a, indicate the region, where subsequent KE resolution measurements were carried out. A typical KE lineout is depicted in Fig. 7, where 10-90 \% intensity transition in the normalized lineout through the sharp edge in the image is equal to $\sim 2.5$ pixels. For the pixel width of $890 \mathrm{~nm}$, it yields $2.2 \mu \mathrm{m}$ Rayleigh resolution. The value of resolution was assessed statistically, based on 10 independent measurements, resulting in half-pitch spatial resolution equal to $r_{\mathrm{KE}}=1.1 \pm 0.2 \mu \mathrm{m}$.

\section{Discussion of the results}

The theoretical half-pitch resolution of the microscope can be expressed as $r_{\mathrm{KE}}=k \lambda /\left(2 \mathrm{NA}_{\mathrm{O}}\right.$ in $)$, where $k$ is illumination and resolution test specific dependent constant [39], for incoherent illumination $(k=0.61)$ this resolution is equal to $18.3 \mathrm{~nm}$ at assumed $\lambda=3 \mathrm{~nm}$ (peak transmission of the Ti filter, just near the absorption edge), so it is much better than measured half-pitch resolution of $0.9 \mu \mathrm{m}$ in the best case (statistically mean value is $1.1 \mu \mathrm{m}$ ). However, the Wolter objective is not a full aperture optic, in fact has a central beam block forming a thin, annular shaped entrance aperture, with outer radius $r_{\mathrm{o}}=7.5 \mathrm{~mm}$ and inner radius $r_{\mathrm{i}}=7 \mathrm{~mm}$, with obscuration ratio $c$ equal to $c=$ $r_{\mathrm{i}} / r_{\mathrm{O}}=0.93$. This leads to a different shape of a point spread function (PSF), which even though has a smaller central lobe, as can be seen in Fig. 8a, but it still has much more pronounced secondary lobes, which extend to a few hundreds of $\mathrm{nm}$ from the center, carrying out much more energy than comparing to a little wider PSF function for a
Fig. 6 SXR images of the objects $(\mathbf{a}, \mathbf{b})$, depicted in Fig. 5, obtained in the "waterwindow" spectral region. Dotted line indicates region where a lineout was made to assess spatial resolution based on the KE test
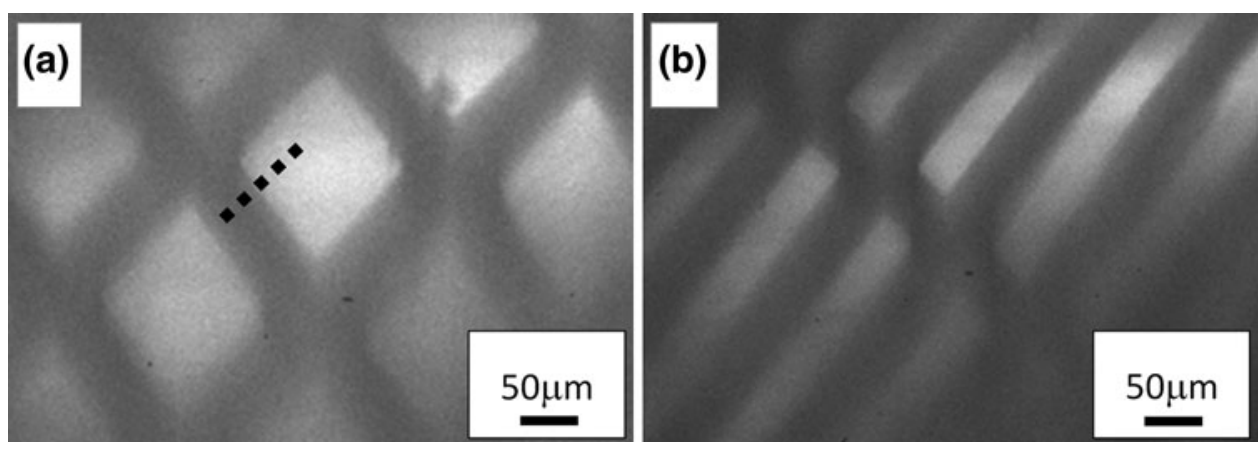


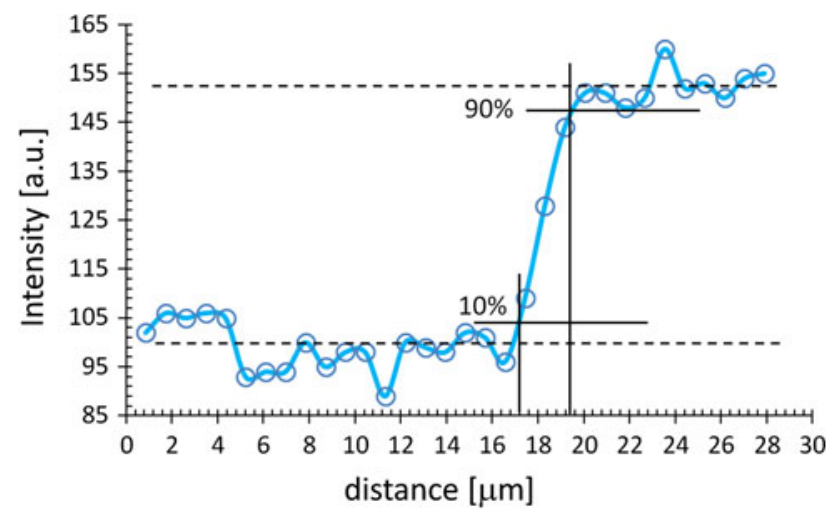

Fig. 7 (color online) KE resolution test result showing Rayleigh resolution equal to 2.5 pixels $=2.2 \mu \mathrm{m}$ or half-pitch spatial resolution equal to $1.1 \mu \mathrm{m}$

full, un-obscured entrance aperture hypothetical objective. Figure $8 \mathrm{~b}$ shows corresponding knife edge functions (KEF) for previously described two types of apertures, calculated based on their PSF functions. A half-pitch spatial resolution for un-obscured aperture, which in our case will be a theoretical resolution limit, is equal to $\sim 18.5 \mathrm{~nm}$. For annular entrance aperture, with $c=0.93$, the expected half-pitch resolution is much worse, equal to $304 \mathrm{~nm}$, which is more than $16 \times$ the diffraction limit. Blue solid line with data points shows the experimentally obtained $\mathrm{KEF}$, from which the half-pitch resolution of $1.1 \mu \mathrm{m}$ was obtained, with lower, 1.b. (900 nm) and upper u.b. $(1.3 \mu \mathrm{m})$ bound on the resolution from statistical measurements; gray and black solid lines, respectively.

Second parasitic parameter that influences the spatial resolution, which was previously studied in EUV microscopy and reported in [10], is a thickness of the object. Owing to the fact that sometimes the thickness of the object is much larger than the depth of focus (DOF), it causes the resolution to be much worse, that for the case of "thin" object. The DOF, defined usually for $20 \%$ intensity decrease, can be calculated for a full aperture objective using DOF $=$ $\pm \lambda /\left(2 \mathrm{NA}_{\mathrm{O} \_ \text {in }}^{2}\right)$ and assuming $\lambda=3 \mathrm{~nm}$, is equal to $\pm 600 \mathrm{~nm}$, or $1.2 \mu \mathrm{m}$ in total, for $50 \%$ intensity drop, however, it was calculated to be $2.09 \mu \mathrm{m}$. For objective, with obscuration ratio $c=0.93$, and $50 \%$ intensity drop, the DOF is much larger and equal to $30.15 \mu \mathrm{m}$. Thus, due to the fact that the thickness of the test objects, $d=13 \mu \mathrm{m}$, is much smaller than the DOF, so its influence on spatial resolution was assumed to be negligible.

Another resolution decreasing factor is the influence of modulation transfer function (MTF) of the CCD detector with relatively large equivalent pixel size of $890 \times 890 \mathrm{~nm}^{2}$ in compared to measured spatial resolution. PSF of the CCD detector in this case will additionally widen computed PSF and KEF for the optical system. Convolution of these two parameters yields the expected half-pitch resolution to be equal to $\sim 480 \mathrm{~nm}$ and corresponding KE function (KEF) can be seen in Fig. $8 \mathrm{~b}$ as a solid violet line.

Additional discrepancy is probably due to optical quality of the Wolter objective mirrors, surface curvature errors, resulting in various aberrations and finally surface roughness of the two-mirror Wolter objective optical system. Particularly, the later might have a noticeable contribution for large $\sigma / \lambda$ ( $\sigma-\mathrm{rms}$ roughness of the mirror), especially for such a short wavelength $\lambda=3 \mathrm{~nm}$, where only a fraction of the total energy resides in the specular beam, described by functions obtained from diffraction calculations and presented in Fig. 8a, while the remaining energy is contained in scattered components [42]. More detailed calculations of the contribution of this effect are under way.

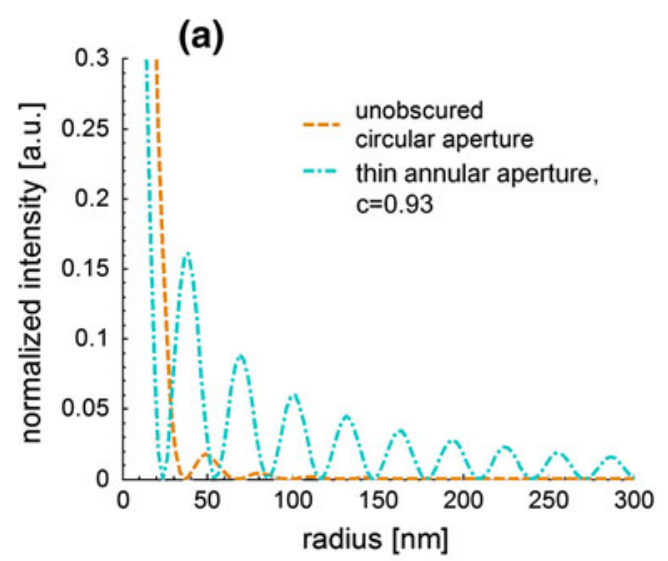

Fig. 8 (color online) Calculated PSF functions (a) and KE functions (b) for un-obscured, full aperture hypothetical objective (dashed line), objective with central beam block (thin annular entrance aperture), for $c=0.93$ (dash-dotted line), such as Wolter type I objective used in

(b)

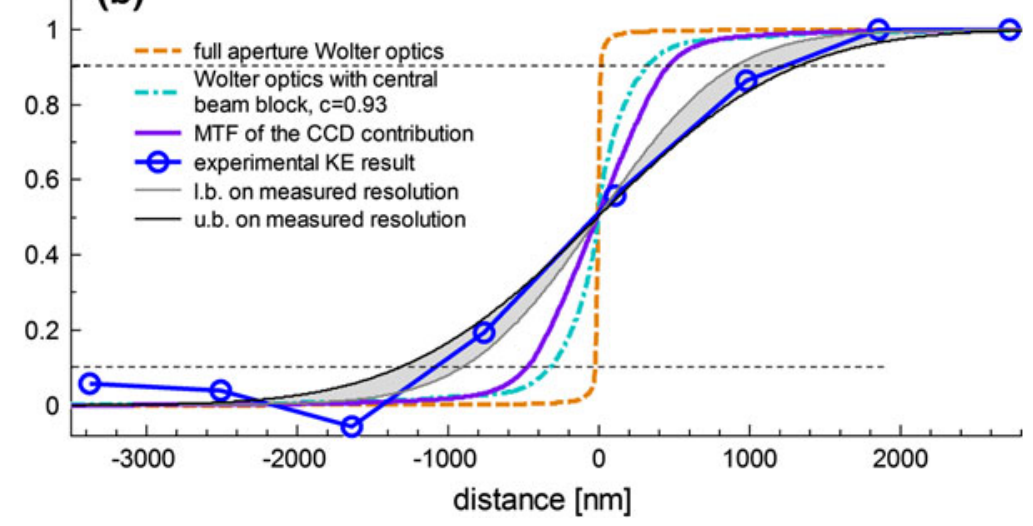

the experiment. In addition in $\mathbf{b}$ MTF contribution of the CCD detector-violet solid line, $\mathrm{KE}$ function obtained experimentally (solid line with circular markers) and statistically measured u.b. and 1.b. on KE resolution (indicated with grey area) 


\section{Conclusions}

We reported on "water-window" compact, desktop microscopy system, employing a laser-plasma SXR source based on a double-stream gas-puff target and a Wolter type I objective. This system allows capturing magnified images of the objects, with magnification of $\sim 15 \times, \sim 1 \mu \mathrm{m}$ halfpitch spatial resolution and exposure time as low as $5 \mathrm{~s}$. We presented a detailed characterization and optimization of both the source and the microscope setup.

The resolution of this system is still quite unsatisfactory for many applications. The system, however, offers imaging capability in the "water-window" spectral range, combining its desktop size, accessibility and simple operation. The lack of dispersion of the condenser and objective and the possibility to change spectral emission of the source by changing the working gas or just the filters allows to perform imaging in other wavelength ranges, for example 18-60 nm using Al filter. This opens new possibilities to exploit different spectral information, now possible to obtain from investigated objects at various wavelengths and allow in turn to study the samples more thoroughly.

Future plans include improvement of the magnification and the spatial resolution of this system by changing the objective to a Fresnel zone plate (ZP), which offers spectral selectivity as well as much higher magnifications and spatial resolution, however, for the price of higher exposures. We also plan to change from argon to nitrogen plasma, where the emission is quasi-monochromaticmuch more suitable for high dispersion $\mathrm{ZP}$ objectives [37].

Acknowledgments The research was supported by the Ministry of Science and Higher Education of Poland and the European Commission's Seventh Framework Program COST Action MP0601, decision number 816/N-COST/2012/0, European Economic Area (EEA) grants and by the National Centre for Science, award number DEC-2011/03/D/ST2/00296. We thank Prof. L. Pina for his help in the preparation of SXR condenser optics. We would also like to thank to René Hudec from Astronomical Institute Academy of Sciences of the Czech Republic for Wolter objective used in this work.

Open Access This article is distributed under the terms of the Creative Commons Attribution License which permits any use, distribution, and reproduction in any medium, provided the original author(s) and the source are credited.

\section{References}

1. D.S. DiCicco, D. Kim, R. Rosser, S. Suckewer, Opt. Lett. 17(2), 157 (1992)

2. L.B. Da Silva, J.E. Trebes, S. Mrowka, T.W. Barbee Jr, J. Brase, J.A. Koch, R.A. London, B.J. MacGowan, D.L. Matthews, D. Minyard, G. Stone, T. Yorkey, E. Anderson, D.T. Attwood, D. Kern, Opt. Lett. 17, 754 (1992)
3. M. Wieland, C. Spielmann, U. Kleineberg, T. Westerwalbesloh, U. Heinzmann, T. Wilhein, Ultramicroscopy 102, 93 (2005)

4. M. Kishimoto, M. Tanaka, R. Tai, K. Sukegawa, M. Kado, N. Hasegawa, H. Tang, T. Kawachi, P. Lu, K. Nagashima, H. Daido, Y. Kato, K. Nagai, H. Takenaka, J. Phys. IV 104, 141 (2003)

5. I.A. Artioukov, A.V. Vinogradov, V.E. Asadchikov, Y.S. Kasyanov, R.V. Serov, A.I. Fedorenko, V.V. Kondratenko, S.A. Yulin, Opt. Lett. 20, 2451 (1995)

6. G. Vaschenko, F. Brizuela, C. Brewer, M. Grisham, H. Mancini, C.S. Menoni, M.C. Marconi, J.J. Rocca, W. Chao, J.A. Liddle, E.H. Anderson, D.T. Attwood, A.V. Vinogradov, I.A. Artioukov, Y.P. Pershyn, V.V. Kondratenko, Opt. Lett. 30(16), 2095 (2005)

7. G. Vaschenko, C. Brewer, F. Brizuela, Y. Wang, M.A. Larotonda, B.M. Luther, M.C. Marconi, J.J. Rocca, C.S. Menoni, Opt. Lett. 31(9), 1214 (2006)

8. P.W. Wachulak, A. Bartnik, H. Fiedorowicz, T. Feigl, R. Jarocki, J. Kostecki, R. Rakowski, P. Rudawski, M. Sawicka, M. Szczurek, A. Szczurek, Z. Zawadzki, Appl. Phys. B 100(3), 461-469 (2010)

9. P.W. Wachulak, A. Bartnik, H. Fiedorowicz, Opt. Lett. 35(14), 2337-2339 (2010)

10. P.W. Wachulak, A. Bartnik, H. Fiedorowicz, J. Kostecki, Opt. Express 19(10), 9541-9550 (2011)

11. K.W. Kim, Y. Kwon, K.Y. Nam, J.H. Lim, K.G. Kim, K.S. Chon, B.H. Kim, D.E. Kim, J.G. Kim, B.N. Ahn, H.J. Shin, S. Rah, K.H. Kim, J.S. Chae, D.G. Gweon, D.W. Kang, S.H. Kang, J.Y. Min, K.S. Choi, S.E. Yoon, E.A. Kim, Y. Namba, K.H. Yoon, Phys. Med. Biol. 51, N99-N107 (2006)

12. M. Berglund, L. Rymell, M. Peuker, T. Wilhein, H.M. Hertz, J. Microsc. 197(pt.3), 268 (2000)

13. G.A. Johansson, A. Holmberg, H.M. Hertz, M. Berglund, Rev. Sci. Instrum. 73(3), 1193 (2002)

14. P.A.C. Takman, H. Stollberg, G.A. Johansson, A. Holmberg, M. Lindblom, H.M. Hertz, J. Microsc. 226(2), 175-181 (2007)

15. M. Bertilson, O. von Hofsten, U. Vogt, A. Holmberg, H.M. Hertz, Opt. Express 17(13), 11057 (2009)

16. K. Murakami, T. Oshino, H. Nakamura, M. Ohtani, H. Nagata, Appl. Opt. 32(34), 7057 (1993)

17. W. Chao, J. Kim, S. Rekawa, P. Fischer, E.H. Anderson, Opt. Express 17(20), 17699 (2009)

18. S. Rehbein, S. Heim, P. Guttmann, S. Werner, G. Schneider, Phys. Rev. Lett. 103, 110801 (2009)

19. B.L. Mesler, P. Fischer, W. Chao, E.H. Anderson, D.H. Kim, J. Vac. Sci. Technol. B 25, 2598 (2007)

20. P. Fischer, D.H. Kim, B.L. Mesler, W. Chao, E.H. Anderson, J. Magn. Magn. Mater. 310(2), 2689 (2007)

21. P. Fischer, D.H. Kim, W. Chao, J.A. Liddle, E.H. Anderson, D.T. Attwood, Mater. Today 9(1-2), 26 (2006)

22. D.H. Kim, P. Fischer, W. Chao, E. Anderson, M.Y. Im, S.Ch. Shin, S.B. Choe, J. Appl. Phys. 99, $08 \mathrm{H} 303$ (2006)

23. B. Nieman, D. Rudolph, G. Schmahl, Opt. Commun. 12, 160 (1974)

24. B. Nieman, D. Rudolph, G. Schmahl, Appl. Opt. 15, 1883 (1976)

25. G. Schneider, B. Riemann, P. Guttmann, D. Rudolph, G. Schmahl, Synchr. Rad. News 8, 19 (1995)

26. C. Jacobsen, S. Williams, E. Anderson, M.T. Browne, C.J. Buckley, D. Kern, J. Kirz, M. Rivers, X. Zhang, Opt. Commun. 86, 351 (1991)

27. C. Jacobsen, J. Kirz, S. Williams, Ultramicroscopy 47, 55 (1992)

28. J. Kirz, C. Jacobsen, S. Lindaas, S. Williams, X. Zhang, E. Anderson, M. Howells, Soft X-Ray Microscopy at the National Synchrotron Light Source, Published in Synchrotron Radiation in the Biosciences (Oxford Univ. Press, Oxford, 1994), p. 563

29. G. Poletti, F. Orsini, D. Batani, Solid State Phenom. 107, 7-10 (2005)

30. A. C. Cefalas, P. Argitis, Z. Kollia, E. Sarantopoulou, T. W. Ford, A. D. Stead, A. Marranca, C. N. Danson, J. Knott, D. Neely, 
Technical Report RAL-TR-98-007, TMR Large-Scale Facilities Access Programme, National Hellenic Research Foundation, Greece (1998)

31. M. Kado, H. Daido, Y. Yamamoto, K. Shinohara, M. C. Richardson, in Proceedings of 8th International Conference on X-ray Microscopy, IPAP Conference Series 7, pp. 41-43 (2005)

32. G. Schneider, Ultramicroscopy 75, 85-104 (1998)

33. W. Meyer-Ilse, D. Hamamoto, A. Nair, S.A. Lelièvre, G. Denbeaux, L. Johnson, A.L. Pearson, D. Yager, M.A. Legros, C.A. Larabell, J. Microsc. 201(3), 395-403 (2001)

34. J. Maser, A. Osanna, Y. Wang, C. Jacobsen, J. Kirz, S. Spector, B. Winn, D. Tennant, J. Microsc. 197(1), 68-79 (2000)

35. L.B. Da Silva, J.E. Trebes, R. Balhorn, S. Mrowka, E. Anderson, D.T. Attwood, T.W. Barbee Jr, J. Brase, M. Corzett, J. Gray, J.A. Koch, C. Lee, D. Kern, R.A. London, B.J. MacGowan, D.L. Mathews, X-ray laser microscopy with an rat sperm nuclei. Science 258, 269 (1992)
36. H. Fiedorowicz, A. Bartnik, R. Jarocki, J. Kostecki, J. Krzywinski, J. Mikołajczyk, R. Rakowski, A. Szczurek, M. Szczurek, J. Alloys Compd. 401, 99 (2005)

37. P.W. Wachulak, A. Bartnik, H. Fiedorowicz, P. Rudawski, R. Jarocki, J. Kostecki, M. Szczurek, Nucl. Instrum. Methods Phys. Res. Sect. B 268(10), 1692-1700 (2010)

38. H. Wolter, Ann. Physik 10, 286 (1952)

39. J.M. Heck, D.T. Attwood, W. Meyer-Ilse, E.H. Anderson, J. X Ray Sci. Technol. 8, 95 (1998)

40. R. L. Kelly, Atomic and ionic spectrum lines below 2000 Angstroms: hydrogen through krypton, J. Phys. Chem. Ref. Data 16(suppl. 1), 386-412 (1987)

41. D. Attwood, Soft X-Rays and Extreme Ultraviolet Radiation (Cambridge University, Cambridge, England, 1999)

42. J. Harvey, N. Choi, A. Krywonos, G. Peterson, M. Bruner, Opt. Eng. 49(6), 063202 (2010) 